Departamento de Cirugia y Laboratorio de Enfermedades Metabólicas. Hospital Clínico Dr. José Joaquín Aguirre, Facultad de Medicina de la Universidad de Chile. Santiago, Chile.

Conflictos de intereses: nada que declarar.

Recibido el 11 de marzo de 2014, aceptado el 8 de mayo de 2015

Correspondencia a: Dr. Italo Braghetto Departamento de Cirugía, Hospital Clínico Dr. José Joaquín Aguirre. Facultad de Medicina Universidad de Chile. Santos Dumont 999, Santiago, Chile.

Teléfono: 56229788334 italobraghetto@gmail.com

\section{Efecto de la gastrectomía tubular vertical en los niveles de ghrelina plasmática en pacientes obesos}

\author{
ITALO BRAGHETTO, CRISTIAN TALADRIZ, \\ ENRIQUE LANZARINI, CARMEN ROMERO
}

\section{Plasma ghrelin levels in the late postoperative period of vertical sleeve gastrectomy}

Background: Plasma ghrelin levels may decrease after vertical sleeve gastrectomy, probably due to the excision of a large portion of the gastric fundus. It is worth exploring the long term effects of this surgical procedure on ghrelin levels and their associations with changes in body weight. Aim: To assess ghrelin levels and changes in body weight, one and five years after a vertical sleeve gastrectomy. Material and Methods: Plasma ghrelin was measured prior to gastric sleeve gastrectomy, immediately after surgery and BMI was controlled at one and five years of the postoperative period in 16 and 10 obese patients, respectively. Mean baseline body mass index was $40.4 \pm 6 \mathrm{~kg} / \mathrm{m}^{2}$. Results: Ghrelin decreased in 10 patients and increased in six during the long term postoperative period. At one year, mean body mass index decreased to $25.1 \pm 1.6 \mathrm{~kg} / \mathrm{m}^{2}$. Changes in weight and ghrelin levels had no relationship whatsoever. At five years of follow up, body mass index had increased to $29.9 \pm 2.5 \mathrm{~kg} / \mathrm{m}^{2}$, again without any association with ghrelin levels. Conclusions: Ghrelin levels may decrease or increase after vertical sleeve gastrectomy and have no association with changes in body weight.

(Rev Med Chile 2015; 143: 864-869)

Key words: Body mass index; Follow-up studies; Gastrectomy; Ghrelin; Laparoscopy.

\section{L} a gastrectomía tubular vertical (GTV) es un procedimiento quirúrgico restrictivo que consiste en la resección del fondo y parte del cuerpo y antro gástrico, logrando una disminución de $90 \%$ de la capacidad de éste ${ }^{1-3}$. Diferentes estudios han demostrado pérdidas del exceso de peso posterior a la GTV entre 51 y $83 \%$ a los 12 meses, con mejoría en co-morbilidades como diabetes, dislipidemia, hipertensión y apnea del sueño comparables a otros procedimientos restrictivos $^{4-7}$. La ghrelina es una hormona gástrica que actúa como regulador del apetito ${ }^{8}$. Se sintetiza principalmente en la mucosa del fondo y cuerpo gástrico, pero también es sintetizada en menor cantidad por el intestino delgado y aún en menor cuantía por el riñón, pulmón, placenta, testículos, ovarios, corazón, páncreas, hipófisis y placenta ${ }^{9-14}$.

Niveles elevados de esta hormona estimulan la ingesta y su secreción comienza a disminuir a medida que la ingesta va aumentando ${ }^{15}$. Hay estudios que han planteado que en los pacientes obesos podría existir una falla en los mecanismos de supresión de liberación de ghrelina ${ }^{16-18}$. Al resecar gran parte del cuerpo y fondo durante la gastrectomía vertical tubular la secreción de ghrelina después de la cirugía podría verse afectada.

En los últimos años se han realizado estudios en relación a los efectos de la GTV sobre los niveles de ghrelina, sin embargo, algunos resultados han sido controversiales, pues mientras algunas revi- 
siones han demostrado disminución en los niveles de ghrelina, otras han visto aumentos de ésta por producción extragástrica de la hormona ${ }^{19-21}$.

El objetivo del presente estudio fue determinar las variaciones de los niveles de ghrelina plasmática en pacientes obesos mórbidos después de la gastrectomía tubular vertical (GTV) y estudiar a largo plazo si los cambios en la curva de peso y del índice de masa corporal (IMC) tienen relación con los niveles de ghrelina postoperatoria.

\section{Pacientes y Métodos}

Se realizó un estudio prospectivo descriptivo, incorporando a 16 pacientes obesos mórbidos, a quienes se les realizó GTV y se les determinaron niveles plasmáticos de ghrelina, junto con el registro del IMC antes y después de un año de la operación.

\section{Obtención de muestras}

Debido a que la ghrelina es una molécula muy inestable, las muestras de sangre se tomaron en tubos de $5 \mathrm{ml}$ con EDTA como anti-coagulante; previamente se agregaron 2.500 Unidades de Aprotinina (Sigma, USA) para los $5 \mathrm{ml}$ de sangre total. Las muestras se trasladaron en hielo inmediatamente al Laboratorio para conservar la cadena de frío.

\section{Determinación de ghrelina activa en muestras de plasma}

Las muestras se centrifugaron a $1.500 \mathrm{~g}$ por 15 min y se guardaron a $4^{\circ} \mathrm{C}$. Las mediciones de ghrelina activa fueron analizadas en duplicado por ELISA, según las instrucciones del proveedor (Linco Research, Missouri, USA). El coeficiente de variación $(\mathrm{CV})$ intra-análisis fue de $3,8 \%$ y la sensibilidad fue de $2,5 \mathrm{fmol} / \mathrm{ml}$ de ghrelina activa. Este ensayo tiene menor porcentaje de reacción cruzada con desacyl-ghrelina. Una vez obtenido el plasma, se agregaron $100 \mu \mathrm{l}$ de $\mathrm{HCl} 1 \mathrm{M}$ por $\mathrm{ml}$ de plasma y se guardó la muestra a $-70^{\circ} \mathrm{C}$ hasta su procesamiento. Las muestras con hemólisis o lipemia fueron rechazadas.

El análisis estadístico utilizado fue la prueba de $t$ de Student para grupos pareados, con un $\mathrm{p}<$ 0,05 . Para el análisis estadístico se hizo una comparación de promedios para muestras pareadas y dio valor $p=0,1981$ para las variaciones de ghrelina en el pre y post operatorio.

\section{Resultados}

En esta serie se incluyen 16 pacientes, ( 9 mujeres y 7 hombres) con una edad promedio de 37,1 $\pm 7,9$ años. En el preoperatorio, el IMC observado fue de $40,4 \pm 6 \mathrm{~kg} / \mathrm{m}^{2}$, llegando a niveles de $25,1 \pm 1,6 \mathrm{~kg} /$ $\mathrm{m}^{2}$ a los 6 meses y $26,9 \pm 2,5$ al año de seguimiento, con un valor $\mathrm{p}$ estadísticamente significativo $(\mathrm{p}<0,05) \cdot \mathrm{Al} 5^{\circ}$ año de seguimiento se observó una re-ganancia de peso en el grupo global hasta un IMC promedio de 29,46 $\pm 3,5 \mathrm{~kg} / \mathrm{m}^{2}$ (Figura 1). En la Figura 2 se muestran los valores de ghrelina plasmática antes y después de la cirugía, en cada uno de los 16 pacientes incluidos en el estudio.

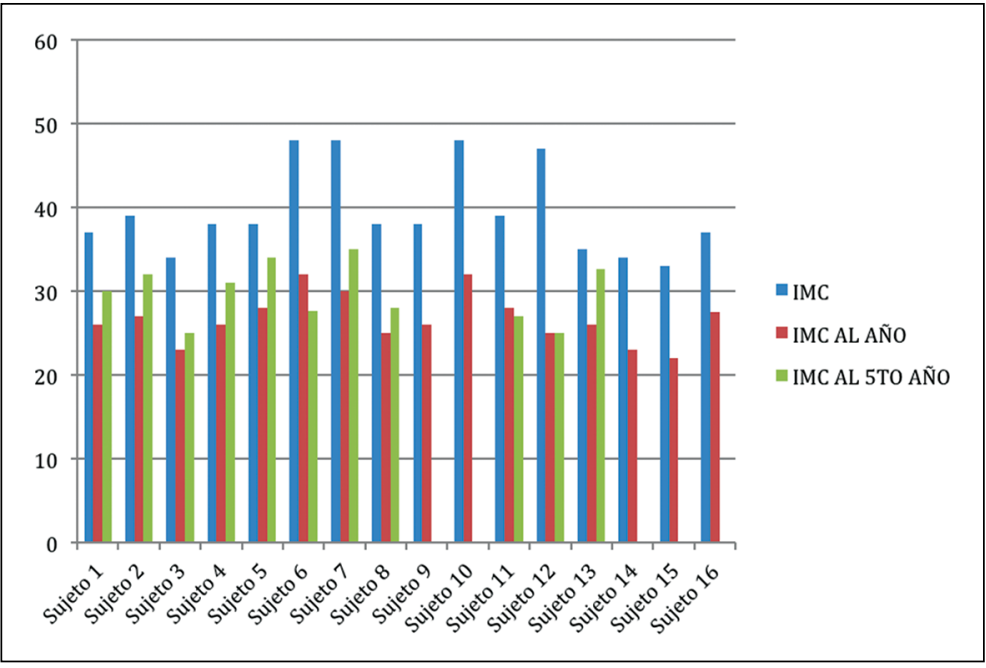

Figura 1. Índices de masa corporal preoperatorio, al año y al $5^{\circ}$ año de seguimiento. 


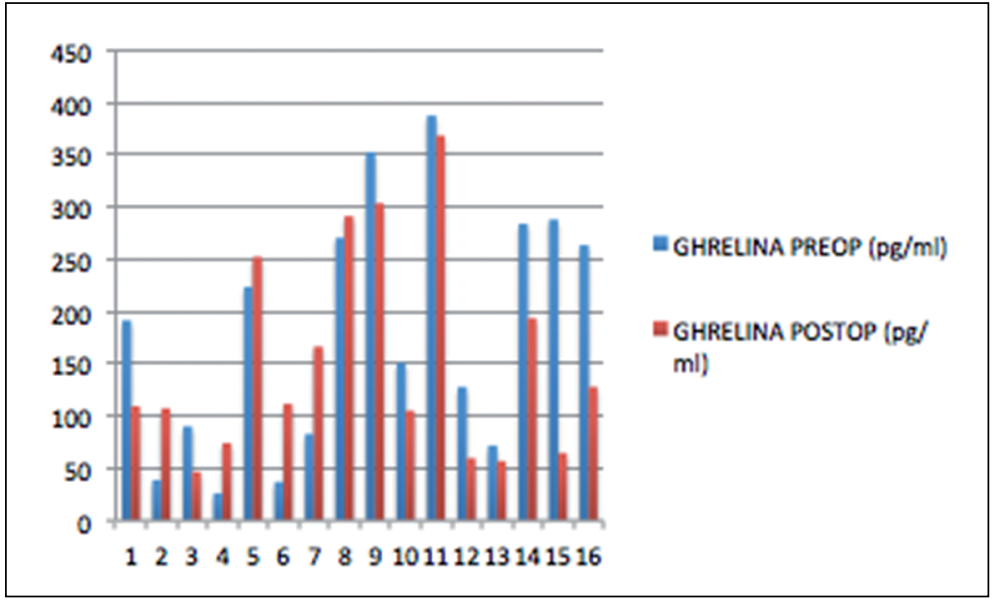

Figura 2. Valores de Ghrelina pre y postoperatoria precoz.
La ghrelina preoperatoria promedio fue de $180,34 \pm 118,23 \mathrm{pg} / \mathrm{ml}$ y la ghrelina postoperatoria promedio en el grupo completo (16 pacientes) fue de $152,41 \pm 100,65 \mathrm{pg} / \mathrm{ml}$ con una variación de $<15,48 \%$. En 10 pacientes $(62,5 \%$ de los sujetos) se observó una disminución significativa de los niveles de ghrelina, en $77,2 \mathrm{pg} / \mathrm{ml}$ promedio $(\mathrm{p}=0,036)$, mientras que los 6 pacientes restantes $(37,5 \%)$ presentaron un aumento en los niveles de ésta en 54,18 pg/ml (Tabla 1 ).
El IMC en el grupo total (16 pacientes) disminuyó de $40,4 \pm 6 \mathrm{~kg} / \mathrm{m}^{2}$ a $25,1 \pm 5 \mathrm{~kg} / \mathrm{m}^{2}$ al primer año, pero se observó recuperación de peso progresiva después del segundo año post cirugía, alcanzando un IMC de 29,9 $\pm 2,46 \mathrm{~kg} / \mathrm{m}^{2}$ al $5^{\circ}$ año de seguimiento (Tabla 2). Al analizar en detalle estos resultados para determinar la relación del comportamiento de IMC y niveles de ghrelina, en los pacientes que presentaron un incremento de los niveles de ghrelina postoperatoria se observó

Tabla 1. Niveles plasmáticos de ghrelina en pacientes obesos sometidos a gastrectomía vertical tubular, comparando valores pre y postoperatorios

\begin{tabular}{|lcccccc|}
\hline Ghrelina & $\mathbf{n}$ & $\mathbf{\%}$ & $\begin{array}{c}\text { Pre operatorio } \\
\text { (promedio } \pm \text { DE) }\end{array}$ & $\begin{array}{c}\text { Post operatorio } \\
\text { (promedio } \pm \text { DE) }\end{array}$ & Delta & $\%$ \\
\hline Disminuye & 10 & 62,5 & $220,75 \pm 110,2$ & $121,25 \pm 92,5^{*}$ & $-77,20$ & $-34,97$ \\
\hline Aumenta & 6 & 37,50 & $113 \pm 106,65$ & $143,55 \pm 111,5$ & 54,18 & 47,94 \\
\hline Grupo total & 16 & 100 & $180,34+118,23$ & $152,41+100,65$ & $-27,93$ & $-15,48$ \\
\hline
\end{tabular}

${ }^{*} \mathrm{p}=0,036 ; \mathrm{DE}=$ desviación estándar.

Tabla 2. Resumen del comportamiento del IMC al $5^{\circ}$ año de seguimiento de acuerdo a los niveles de ghrelina postoperatoria precoz

\begin{tabular}{|lccccc|}
\hline & \multicolumn{1}{c}{$\begin{array}{c}\text { Ghrelina } \\
\text { Preop }\end{array}$} & Postop & $\begin{array}{c}\text { IMC } \\
\text { Preop }\end{array}$ & Postop 1 año & Postop 5 años \\
\hline Grupo total $(n=16)$ & $180,34 \pm 118,2$ & $152,41 \pm 100,65$ & $40,4 \pm 6,0$ & $25,1 \pm 6,1$ & $29,9 \pm 2,46$ \\
\hline Aumento $(n=6)$ & $186,5 \pm 107,9$ & $204,4 \pm 82,1$ & $41,5 \pm 4,6$ & $27,6 \pm 2,3$ & $32,3 \pm 2,7$ \\
\hline Disminución $(n=10)$ & $176,7 \pm 118,0$ & $121,3 \pm 92,5$ & $38,2 \pm 5,0$ & $25,9 \pm 2,8$ & $27,6 \pm 2,5$ \\
\hline
\end{tabular}


Ghrelina post gastrectomía vertical tubular - I. Braghetto et al

Tabla 3. Pacientes que presentaron alzas en los niveles de ghrelina postoperatoria $(n=6)$

\begin{tabular}{|lccccc|}
\hline Observación & IMC & IMC al año & $\mathbf{I M C}$ al $\mathbf{5}^{\mathbf{0}}$ año & Ghrelina preop (pg/ml) & Ghrelina postop (pg/mI) \\
\hline Paciente 2 & 39 & 27 & 32 & 38,65 & 107,26 \\
\hline Paciente 4 & 38 & 26 & 31 & 25,93 & 74,09 \\
\hline Paciente 5 & 38 & 28 & 34 & 223,55 & 252,45 \\
\hline Paciente 6 & 48 & 32 & 27,6 & 36,73 & 111,55 \\
\hline Paciente 7 & 48 & 30 & 35 & 82,64 & 166,4 \\
\hline Paciente 8 & 38 & 25 & 28 & 270,5 & 291,3 \\
\hline Promedio & 41,5 & 28,00 & 31,27 & 113,00 & 167,18 \\
\hline DE* & 5,05 & 2,61 & 3,04 & 106,65 & 87,21 \\
\hline
\end{tabular}

*DE = desviación estándar.

Tabla 4. Pacientes que presentaron disminución en los niveles de ghrelina postoperatoria $(n=10)$

\begin{tabular}{|lccccc|}
\hline Observación & IMC & IMC al año & IMC al $\mathbf{5}^{\circ}$ año & Ghrelina preop (pg/ml) & Ghrelina postop (pg/mI) \\
\hline Paciente 1 & 37 & 26 & 30 & 191,45 & 109,4 \\
\hline Paciente 3 & 34 & 23 & 25 & 90,01 & 46,48 \\
\hline Paciente 9 & 38 & 26 & sin datos & 352,65 & 303,6 \\
\hline Paciente 10 & 48 & 32 & sin datos & 150,9 & 105,08 \\
\hline Paciente 11 & 39 & 28 & 27 & 387,55 & 368,45 \\
\hline Paciente 12 & 47 & 25 & 25 & 127,75 & 59,64 \\
\hline Paciente 13 & 35 & 26 & 32,7 & 71,71 & 56,74 \\
\hline Paciente 14 & 34 & 23 & sin datos & 283,9 & 193,7 \\
\hline Paciente 15 & 33 & 22 & sin datos & 288,05 & 64,51 \\
\hline Paciente 16 & 37 & 27,5 & sin datos & 263,5 & 127,9 \\
\hline Promedio & 38,2 & 25,9 & 26,8 & 220,75 & 143,55 \\
\hline DE* & 5,3 & 2,9 & 2,4 & 110,2 & 111,5 \\
\hline
\end{tabular}

*DE $=$ desviación estándar.

un incremento del IMC al $5^{\circ}$ año comparado al IMC del $1^{\text {er }}$ año de postoperados, con un incremento de 4 puntos, y en los pacientes en los cuales disminuyó la ghrelina plasmática el incremento del IMC al $5^{\circ}$ año fue de 2 puntos en comparación al IMC al primer año, sin que exista por lo tanto una correlación entre los niveles plasmáticos de ghrelina postoperatoria y la recuperación de peso (Tablas 3 y 4 ).

Las variaciones en los niveles de ghrelina no tuvieron significación estadística, probablemente influido por un bajo número de pacientes estudiados.

\section{Discusión}

Varios estudios en la literatura internacional reportan los resultados postoperatorios de los niveles de ghrelina plasmática en pacientes sometidos a gastrectomía vertical tubular, pero ninguno se refiere a su relación con la recuperación o no de peso en un seguimiento prolongado. Algunos estudios recientes observaron una reducción significativa de los niveles plasmáticos de ghrelina hasta 6 meses después de la cirugía. Los niveles de ghrelina se caracterizan por un aumento durante los períodos de ayuno y una rápida caída 
postprandial. En los pacientes con GVT se ha observado permanentemente una disminución de los niveles de ghrelina hasta 1 año después de la cirugía, pero estos valores son ligeramente más altos comparados con los niveles observados después de los 3 meses de operado y curiosamente sin disminución postprandial, lo que pone un grado de interrogación al respecto ${ }^{24,31-35}$. Otros estudios experimentales no han demostrado disminución de los niveles de ghrelina post gastrectomía vertical tubular y la posible explicación es la producción extragástrica de ghrelina ${ }^{23,36}$.

De acuerdo a los resultados del presente estudio, se confirma que la GTV presenta buenos resultados a mediano plazo, con baja de peso comparable con otras publicaciones ${ }^{26-31}$ y se ha constatado la disminución de la liberación de ghrelina secundaria a la resección del fondo gástrico en un importante número de pacientes, lo que no ocurre después de otras operaciones bariátricas en las que no se reseca el fondo gástrico. En un estudio efectuado en nuestro Departamento se demostró también disminución de la ghrelina plasmática después de resección gástrica ${ }^{32}$. A pesar de que en la mayoría de los pacientes se observa una tendencia hacia la baja en los niveles de esta hormona, un porcentaje importante de los pacientes $(37,5 \%)$ presentó una elevación de los niveles de ghrelina.

De los 6 pacientes que presentaron alzas, aquellos que tenían niveles preoperatorios menores a $100 \mathrm{pg} / \mathrm{ml}(\mathrm{n}=4)$ prácticamente duplicaron $\mathrm{y}$ algunos inclusive triplicaron sus valores de ghrelina en el postoperatorio. Esta variación tan importante no se observa en aquellos pacientes que presentaron una disminución de la ghrelina postoperatoria, donde en promedio tuvieron una baja de 75,2 pg/ml. No se logró identificar una asociación entre los valores de ghrelina con el IMC pre o postoperatorio. No tenemos una explicación clara para estos hallazgos. La variabilidad en los valores de la hormona podría verse afectada por el hecho de que no solamente es el estómago el encargado de secretar la ghrelina, sino que existen otros órganos que también inciden en su síntesis ${ }^{9-14}$, por lo que podríamos estar frente a un mecanismo de compensación. Por otra parte, si bien durante el acto quirúrgico se reseca gran parte del cuerpo y fondo gástrico, no hay seguridad de haber resecado toda el área donde se localizan las células liberadoras de la hormona. No existen datos en la literatura respecto de este punto. Ade- más, un reciente estudio sugiere que el tamaño del estómago es probablemente más importante que la disminución de los niveles de ghrelina en la mantención de la baja de peso después de resección gástrica ${ }^{32}$.

Estudios recientes han demostrado incrementos significativos en el apetito de pacientes con cáncer que han sido suplementados con ghrelina exógena por lo que podríamos asumir que obesos que tengan alzas significativas en el postoperatorio de esta hormona podrían tener mayores tasas de falla con re-ganancia de peso y aumento en su IMC en el seguimiento a largo plazo después de la GTV. De acuerdo a lo observado en esta serie, la recuperación del peso a largo plazo no se asociaría con la disminución o elevación de ghrelina postoperatoria.

\section{Referencias}

1. Baltasar A, Serra C, Pérez N, Bou R, Bengochea M, Ferri L. Laparoscopic sleeve gastrectomy: a multipurpose bariatric operation. Obes Surg 2005; 15: 1124-8.

2. Ren CJ, Patterson E, Gagner M. Early results of laparoscopic biliopancreatic diversion with duodenal switch: a case series of 40 consecutive patients. Obes Surg 2000; 10: 514-23.

3. Almogy G, Crookes PF, Anthone GJ. Longitudinal gastrectomy as a treatment for the high risk superobese patient. Obes Surg 2004; 14: 492-7.

4. Mognol P, Chosidow D, Marmuse J. Laparoscopic sleeve gastrectomy as an initial bariatric operation for high-risk patients: initial results in 10 patients. Obes Surg 2005; 15: 1030-3.

5. Lee CM, Cirangle PT, Jossart GH. Vertical gastrectomy for morbid obesity in 216 patients: report of two-year results. Surg Endosc 2007; 21: 1810-6.

6. Himpens J, Dapri G, Cadiere G. A prospective randomized study between laparoscopic gastric banding and laparoscopic isolated sleeve gastrectomy: results after 1 and 3 years. Obes Surg 2006; 16: 1450-6.

7. Braghetto I, Korn O, Valladares H, Gutiérrez L, Csendes A, Debandi A, et al. Laparoscopic sleeve gastrectomy: surgical technique, indications and clinical results. Obes Surg 2007; 17: 1442-50.

8. Kojima M, Hosoda H, Date Y, Nakazato M, Matsuo $\mathrm{H}$, Kangawa K. Ghrelin is a growth hormone releasing acylated peptide from stomach. Nature 1999; 402: 65660.

9. Wang G, Lee HM, Englander E. Ghrelin-not just ano- 
ther stomach hormone. Regul Pept 2002; 105: 75-81.

10. Tena-Sempere M, Barreiro ML, González LC, Gaytan F, Zhang FP, Caminos JE, et al. Novel expression and functional role of ghrelin in rat testis. Endocrinology 2002; 143: 717-25.

11. Gaytan F, Barreiro ML, Chopin LK, Herrington AC, Morales C, Pinilla L, et al. Immunolocalization of ghrelin and its functional receptor, the type la growth hormone secretagogue receptor, in the cyclic human ovary. J Clin Endocrinol Metab 2003; 88: 879-87.

12. Caminos JE, Gualillol O, Lago F, Otero M, Blanco M, Gallego R, et al. The endogenous growth hormone secretagogue (ghrelin) is synthesized and secreted by chondrocytes. Endocrinology 2005; 146: 1285-92.

13. Date $Y$, Nakazato M, Hashiguchi S, Dezaki K, Mondal MS, Hosoda $\mathrm{H}$, et al. Ghrelin is present in pancreatic -cells of humans and rats and stimulates insulin secretion. Diabetes 2002; 51: 124-9.

14. Lai KC, Cheng C, Leung PS. The ghrelin system in acinar cells. Localization, expression, and regulation in the exocrine pancreas. Pancreas 2007; 35: 1-8.

15. Shiiya T, Nakazato M, Mizuta M, Date Y. Plasma ghrelin levels in lean and obese humans and the effect of glucose on secretion. Endocrinol Metab 2002; 87: 240-4.

16. English PJ, Ghatei MA, Malik IA, Bloom SR, Wilding JP. Food fails to suppress ghrelin levels in obese humans. J Clin Endocrinol Metab 2002; 87: 2984-5.

17. Peterli R, Wölnerhanssen B, Peters T, Devaux N, Kern $\mathrm{B}$, Christoffel-Courtin C, et al. Improvement in glucose metabolism after bariatric surgery: comparison of laparoscopic Roux-en-Y gastric bypass and laparoscopic sleeve gastrectomy: a prospective randomized trial. Ann Surg 2009; 250: 234-41.

18. Basso N, Capoccia D, Rizzello M, Abbatini F, Mariani P, Maglio C, et al. First-phase insulin secretion, insulin sensitivity, ghrelin, GLP-1, and PYY changes $72 \mathrm{~h}$ after sleeve gastrectomy in obese diabetic patients: the gastric hypothesis. Surg Endosc 2011; 25: 3540-50.

19. Langer FB, Reza Hoda MA, Bohdjalian A, Felberbauer FX, Zacherl J, Wenzl E, et al. Sleeve gastrectomy and gastric banding: effects on plasma ghrelin levels. Obes Surg 2005; 15: 1024-9.

20. Dimitriadis E, Daskalakis M, Kampa M, Peppe A, Papadakis JA, Melissas J. Alterations in gut hormones after laparoscopic sleeve gastrectomy: a prospective clinical and laboratory investigational study. Ann Surg 2013; 257: 647-54.

21. Sabench F, Hernández M, Feliu A, Blanco S, Morandeira A, del Castillo D. Influence of Sleeve Gastrectomy on Several Experimental Models of Obesity: Metabolic and
Hormonal Implications. Obes Surg 2008; 18: 97-108.

22. Frezza EE, Reddy S, Gee LL, Wachtel MS. Complications after sleeve gastrectomy for morbid obesity. Obes Surg 2009; 19: 684-7.

23. Fuks D, Verhaeghe P, Brehant O, Sabbagh C, Dumont F, Riboulot M, et al. Results of laparoscopic sleeve gastrectomy: a prospective study in 135 patients with morbid obesity. Surgery 2009; 145: 106-13.

24. Gan SS, Talbot ML, Jorgensen JO. Efficacy of surgery in the management of obesity-related type 2 diabetes mellitus. ANZ J Surg 2007; 77: 958-62.

25. Hakeam HA, O'Regan PJ, Salem AM, Bamehriz FY, Jomaa LF. Inhibition of C-reactive protein in morbidly obese patients after laparoscopic sleeve gastrectomy. Obes Surg 2009; 19 (4): 456-60.

26. Himpens J, Dapri G, Cadiere GB. A prospective randomized study between laparoscopic gastric banding and laparoscopic isolated sleeve gastrectomy: results after 1 and 3 years. Obes Surg 2006; 16: 1450-6.

27. Karamanakos SN, Vagenas K, Kalfarentzos F, Alexandrides TJ. Weight loss, appetite suppression, and changes in fasting and postprandial ghrelin and peptide-YY levels after Roux-en-Y gastric bypass and sleeve gastrectomy: a prospective, double blind study. Ann Surg 2008; 247: 401-7.

28. Hady R, Golaszewski P, Zbucki RL, Dadan J. The influence of laparoscopic adjustable gastric banding and laparoscopic sleeve gastrectomy on weight loss, plasma ghrelin, insulin, glucose and lipids. Folia Histochemica et Cytobiologica 2012; 50: 292-303.

29. Lundholm K, Gunnebo L, Körner U, Iresjö BM, Engström C, Hyltander A, et al. Effects by daily long term provision of ghrelin to unselected weight-losing cancer patients: a randomized double-blind study. Cancer 2010; 15; 116: 2044-52.

30. Peterli R, Steinert RE, Woelnerhanssen B, Peters T, Christoffel-Courtin C, Gass M, et al. Metabolic and Hormonal Changes After Laparoscopic Roux-en-Y Gastric Bypass and Sleeve Gastrectomy: a Randomized, Prospective Trial. Obes Surg 2012; 22: 740-8.

31. Jiménez A, Casamitjana R, Flores L, Viaplana J, Corcelles R, Lacy A, et al. Long-term effects of sleeve gastrectomy and Roux-en-Y gastric bypass surgery on type 2 diabetes mellitus in morbidly obese subjects. Ann Surg 2012; 256: 1023-9.

32. Carrasco F, Rojas P, Csendes A, Codoceo J, Inostroza J, Basfifer $\mathrm{K}$, et al. Changes in ghrelin concentrations one year after resective and non-resective gastric bypass: associations with weight loss and energy and macronutrient intakes. Nutrition 2012; 28: 757-61. 